\title{
Intervenolin suppresses gastric cancer cell growth through the induction of TSP-1 secretion from fibroblast-like stromal cells
}

\author{
JUNJIRO YOSHIDA, HIKARU ABE, TAKUMI WATANABE and MANABU KAWADA
}

Institute of Microbial Chemistry (BIKAKEN), Microbial Chemistry Research Foundation, Tokyo 141-0021, Japan

Received April 24, 2018; Accepted September 17, 2018

DOI: $10.3892 / \mathrm{ol} .2018 .9485$

\begin{abstract}
Fibroblast-like stromal cells modulate the growth of cancer cells, both positively and negatively. Growth modulation is achieved through the secretion of regulatory factors as well as by proteins within the extracellular matrix. Those cellular interactions present attractive targets for cancer chemotherapy. It was demonsrated a novel natural compound, intervenolin (ITV), inhibited the in vitro growth of human gastric cancer cells when co-cultured with stromal cells. Importantly, the inhibition was enhanced by the presence of stromal cells. The present study reported a mechanism of ITV action. Human gastric fibroblast-like stromal cells (Hs738) were treated with ITV. The resultant conditioned medium (ITV CM) inhibited the growth of human gastric cancer cells and suppressed the level of c-Myc protein. This result suggested that ITV negatively modulated cancer cell growth by upregulating the secretion of factors originating from stromal cells in the co-culture system. To better understand the mechanism, ITV CM was subjected to proteomic analysis. The data revealed that one of the candidate regulators was thrombospondin-1 (TSP-1). Recombinant human TSP-1 protein inhibited the growth of gastric cancer cells. Moreover, the growth-inhibitory activities of ITV CM as well as that of recombinant TSP-1 were blocked by neutralizing antibody targeting TSP-1. These results suggested that ITV inhibited the growth of gastric cancer cells through its modulation of stromal cell function.
\end{abstract}

Correspondence to: Dr Manabu Kawada, Institute of Microbial Chemistry (BIKAKEN), Microbial Chemistry Research Foundation, 3-14-23 Kamiosaki, Shinagawa, Tokyo 141-0021, Japan

E-mail:kawadam@bikaken.or.jp

Abbreviations: ITV, intervenolin; CM, conditioned media; ITV CM, the CM taken from human gastric fibroblast-like Hs738 cells treated with ITV; Ctrl CM, CM from non-treated-Hs738; TSP-1, thrombospondin-1; stromal cells, fibroblast-like stromal cells; $\alpha$-SMA, $\alpha$-smooth muscle actin

Key words: cancer chemotherapy, natural compound, tumor-stromal cell interactions, fibroblast-like stromal cells, co-culture, gastric cancer, thrombospondin-1, c-Myc

\section{Introduction}

Tumor tissue contains not only cancer cells but also several stromal components, such as macrophages, immune cells and fibroblasts. Those cells that are profoundly involved in the regulation of cancer cell growth. Many studies have reported that fibroblasts exert important effects on cancer cell growth. Fibroblast-like stromal cells (stromal cells) contained in tumor tissues exhibit a myofibroblast phenotype (expressing $\alpha$-smooth muscle actin). This population regulates cancer cell growth (1), cancer cell stemness $(2,3)$, formation of a premetastatic niches (4) and drug resistance $(5,6)$, through the secretion of soluble factors. Thus, interactions between cancer cells and stromal cells (tumor-stromal cell interactions) are attractive targets for cancer therapy. Indeed, many studies have reported the presence of positive regulatory interactions between cancer cells and stromal cells mediated by secreted factors. However, in a mouse model, others have shown that stromal cells can have a negative regulatory impact in a mouse model. Yin et al (7), reported that IL-25 secreted from tumor-associated fibroblasts suppressed mammary tumor metastasis and that IL-25 secretion was increased by a lignin derivative.

We have focused on the negative regulation of cancer cells by stromal cell secreted factors. This approach offers a novel strategies for discovering new cancer therapeutics. We used a co-culture system to screen small molecules from natural sources such as microbial culture media, seeking molecules that modulated tumor-stromal cell interactions. In additional studies, we used co-culture systems pairing cancer cells and stromal cells from several organs. We reported the identification of small molecules that suppressed cancer cell growth through modulation of stromal cells. Leucinostatin A and Phthoxazolin A were found by co-culture screening of prostate cancer cells with prostate stromal cells. Those agents suppressed cancer cell growth by inhibiting the expression of insulin-like growth factor-1 (IGF-1) by stromal cells $(8,9)$. We also reported that MEK-inhibitor I induced the secretion of GAPDH by gastric stromal cells, a process that suppressed gastric cancer cell growth (10). In a recent study, we found a novel compound, intervenolin (ITV), from the culture medium of Nocardia sp. M196-86F2 (11). ITV inhibited the in vitro growth of human gastric cancer cells. Importantly, inhibition was greater when the cancer cells were co-cultured with stromal cells. Based on this result, we carried out proteomic analysis of conditioned 
medium from human gastric fibroblast-like stromal cells (Hs738), and the results showed that ITV induced TSP-1 secretion from Hs738 cells.

TSP-1 is a glycoprotein that forms a homo tetramer in the extracellular microenvironment. It is secreted by several cell types, including platelets, epithelial cells and fibroblasts. Rodríguez-Manzaneque et al (12), reported that TSP-1 showed antitumor activity. In the present study, we report that TSP-1 from conditioned medium (CM) of Hs738 cells treated with ITV inhibited the growth of cancer cells through its TSP-1 receptor.

\section{Materials and methods}

Cell lines and reagents. Human gastric cancer cell lines MKN-7 and MKN-74 were obtained from the RIKEN cell bank (Tsukuba, Japan). MKN-7 and MKN-74 stably express a transfected GFP vector as described (10). Cancer cell lines were maintained in Dulbecco's Modified Eagle's Medium (DMEM; Nissui, Tokyo, Japan) supplemented with $10 \%$ fetal bovine serum (FBS; Sigma-Aldrich; Merck KGaA, Darmstadt, Germany), $100 \mathrm{U} / \mathrm{ml}$ penicillin G (Thermo Fisher Scientific, Inc., Waltham, MA, USA), and $100 \mu \mathrm{g} / \mathrm{ml}$ streptomycin (Thermo Fisher Scientific, Inc.) at $37^{\circ} \mathrm{C}$ with $5 \% \mathrm{CO}_{2}$. Hs 738 . St/Int (Hs738) human gastric stromal cells (CRL-7869) were obtained from the ATCC (American Type Culture Collection, Manassas, VA, USA). Stromal cells were maintained in DMEM supplemented with $10 \% \mathrm{FBS}, 100 \mathrm{U} / \mathrm{ml}$ penicillin $\mathrm{G}, 100 \mu \mathrm{g} / \mathrm{ml}$ streptomycin, ITH $(5 \mu \mathrm{g} / \mathrm{ml}$ insulin, $5 \mu \mathrm{g} / \mathrm{ml}$ transferrin, and $1.4 \mu \mathrm{M}$ hydrocortisone), and $5 \mathrm{ng} / \mathrm{ml}$ basic FGF (PeproTech, Inc., Rocky Hill, NJ, USA) at $37^{\circ} \mathrm{C}$ with 5\% $\mathrm{CO}_{2}$ as described (10). Recombinant human thrombospondin-1 protein (3074-TH) was purchased from R\&D Systems, Inc., (Minneapolis, MN, USA). Neutralizing antibodies for TSP-1, A4.1 (mouse monoclonal, MS-418-PABX) and C6.7 (mouse monoclonal, MS-420-PABX) were purchased from NeoMarkers, Inc., (Fremont, CA, USA). ITV was synthesized as described previously (11).

Preparation of CM from Hs738. Hs738 cells were cultured at $5 \times 10^{4}$ cells $/ \mathrm{ml}$ in DMEM supplemented with ITH and $5 \%$ FBS. After 1 day, the medium was replaced with fresh medium including ITV $(0.25 \mu \mathrm{g} / \mathrm{ml})$ without FBS (serum-free $\mathrm{CM})$. After an additional 4 days of incubation, $\mathrm{CM}$ from Hs738 (Ctrl CM) or ITV-treated Hs738 (ITV CM) were collected and centrifuged to remove debris. Gastric cancer cells $\left(3 \times 10^{5}\right.$ cells $\left./ \mathrm{ml}\right)$ were inoculated in $1 \mathrm{ml}$ of the $75 \%$ $\mathrm{CM}$ of Hs738 cells or assay medium alone in $35-\mathrm{mm}$ dishes and cultured for 1 day with dialyzed FBS. The cells were washed with phosphate-buffered saline (PBS) and the cell lysates were prepared for western blotting. For cell growth assays, MKN-74 cells were cultured at $5 \times 10^{3}$ cells/well in 96-well plates in Hs738 CM at the indicated concentration with dialyzed FBS.

SDS-PAGE and proteomic analysis of CM. Serum-free CMs were collected from Hs738 cells treated with or without ITV. Proteins were separated by SDS-PAGE, and the gel was stained with a PlusOne silver staining kit (GE Healthcare, Little Chalfont, UK) according to the manual. Bands showing different patterns in CMs treated with and without ITV were excised. These excised bands were digested with trypsin, and the digested peptides were then subjected to LC-MS/MS analysis (LTQ-Orbitrap; Thermo Fisher Scientific, Inc.). For tryptic in-gel digestion, the candidate protein bands were cut into small pieces and destained with $100 \mu \mathrm{L}$ of a 1:1 solution of $30 \mathrm{mM}$ potassium ferricyanide (Fujifilm Wako Pure Chemical Industries, Ltd., Tokyo, Japan) and $100 \mathrm{mM}$ sodium thiosulfate (Fujifilm Wako Pure Chemical Industries, Ltd.). Reduction was performed by re-swelling the gel pieces in $100 \mu \mathrm{L}$ of $10 \mathrm{mM}$ dithiothreitol (Nacalai Tesque, Tokyo, Japan) in $100 \mathrm{mM} \mathrm{NH}_{4} \mathrm{HCO}_{3}$ (Nacalai Tesque) buffer solution and incubated at $56^{\circ} \mathrm{C}$ for $1 \mathrm{~h}$. After cooling, the excess liquid was removed and quickly replaced by the same volume of freshly prepared $55 \mathrm{mM}$ iodoacetamide in $100 \mathrm{mM} \mathrm{NH} \mathrm{HCO}_{3}$ solution. The iodoacetamide solution was removed and the gel pieces were washed with $200 \mu \mathrm{L}$ $50 \%$ acetonitrite $(\mathrm{ACN})$ in $100 \mathrm{mM} \mathrm{NH} \mathrm{HCO}_{3}$ solution for $10 \mathrm{~min}$, and the liquid was removed. One band (gel piece) required 15-20 $\mu \mathrm{L}$ of $0.01 \mu \mathrm{g}$ trypsin (Trypsin Gold, Mass Spectrometry Grade; Promega Corporation, Madison, WI, USA) in $100 \mathrm{mM} \mathrm{NH} \mathrm{HCO}_{3}$ digestion buffer. After incubating the reaction mixture at $37^{\circ} \mathrm{C}$ overnight, the digestion buffer was removed and saved in a new tube. The peptides from the gel pieces were extracted by adding $15-20 \mu \mathrm{L}$ of $50-95 \% \mathrm{ACN}$ in $0.1 \%$ trifluoroacetic acid (TFA) aq. and incubating for $15 \mathrm{~min}$ with sonication. Finally, the samples were purified using Solid Phase Extraction C-TIP (Nikkyo Technos Co., Ltd., Tokyo, Japan) after adding $25 \mu \mathrm{L}$ of $2 \%$ $\mathrm{ACN}$ in $0.1 \%$ TFA aq. Samples were kept in $-20^{\circ} \mathrm{C}$ before being analyzed by LC-MS/MS.

LC-ESI MS/MS analysis. LC-ESI MS/MS analysis was performed using a Paradigm MS4 LC system (Michrom BioResources, Auburn, CA, USA) coupled with an LTQ Orbitrap $^{\mathrm{TM}}$ XL mass spectrometer (Thermo Fisher Scientific, Inc.). An L-column ODS, $3 \mu \mathrm{m}, 0.1 \mathrm{~mm}$ id x $50 \mathrm{~mm}$ (CERI, Tokyo, JAPAN) was used. HPLC was performed using a linear gradient solvent system (solvent A; $0.1 \%$ formic acid in $2 \% \mathrm{ACN}$ aq., solvent $\mathrm{B} ; 0.1 \%$ formic acid in $90 \% \mathrm{ACN}$ ) from 5 to $45 \%$ solvent $\mathrm{A}$ at a flow rate of $0.5 \mu \mathrm{L} / \mathrm{min}$ for $20 \mathrm{~min}$ followed by MS/MS equipped with a CaptiveSpray ${ }^{\mathrm{TM}}$ source (Bruker Corporation, Billerica, MA, USA). The sprayer voltage was set to $1.8 \mathrm{kV}$, and capillary temperature was set to $200^{\circ} \mathrm{C}$. Parent mass peaks with ranges from $\mathrm{m} / \mathrm{z} 400$ to 2,000 were selected for MS/MS analysis by Data Dependent Top3. Normalized collision energy was fixed at 35 . The MS/MS data were processed using Xcalibur ${ }^{\mathrm{TM}} 2.2$ SP1.48. Peptides were searched with MASCOT Server 2.5 (Matrix Science, Inc., Boston, MA, USA). The search parameters were set as follows: cRAP 20100518 (112 sequences; 37,418 residues) and IPI_human (91,464 sequences; 36,355,611 residues) database, enzyme set for trypsin, allowance of one missed cleavage, variable modifications were carbamidomethylation at cysteine residues and oxidation at methionine residues, peptide and MS/MS tolerance was $5 \mathrm{ppm}$ and $0.6 \mathrm{Da}$, respectively, and the limit of peptide charges state was $2+$, and $3+$.

Immunoblotting. Cells $\left(3 \times 10^{5}\right)$ were cultured in 6-well plates for the indicated times. The cells were washed twice with 
ice-cold PBS containing $100 \mu \mathrm{M} \mathrm{Na} \mathrm{VO}_{4}$ and then treated with lysis buffer (20 mM Hepes, $\mathrm{pH} 7.5,150 \mathrm{mM} \mathrm{NaCl}$, $1 \%$ Triton X-100, 10\% glycerol, 1 mM EDTA, $50 \mathrm{mM} \mathrm{NaF}$, $50 \mathrm{mM} \beta$-glycerophosphate, $1 \mathrm{mM} \mathrm{Na} \mathrm{VO}_{4}$, and $25 \mu \mathrm{g} / \mathrm{ml}$ each of antipain, leupeptin, and pepstatin) (13). Equal amounts of protein extracts were separated by SDS-PAGE and transferred onto Immobilon transfer membrane (IPVH304F0, Millipore, Bedford, MA). After incubation with the primary antibody at room temperature (RT) for $1 \mathrm{~h}$, membranes were incubated with HRP-conjugated secondary antibody at RT, $30 \mathrm{~min}$. ECL Western Blotting Detection Reagents (RPN2209; GE Healthcare) were used to visualize the immunoblot signals and signals were determined by ImageQuant LAS4000 mini (GE Healthcare). Relative intensities of determined bans were calculated as peak area using Image $\mathbf{J}$ (National Institutes of Health, Bethesda, MD, USA). The following antibodies from Santa Cruz Biotechnology, Inc., (Dallas, TX, USA) were used for primary labeling: Anti-TSP-1 (1:1,000; mouse monoclonal, sc-59887), anti-CDK4 (1:1,000; rabbit polyclonal, sc-260) and anti-Rb (1:1,000; rabbit polyclonal, sc-50). Anti-c-Myc antibody $(1: 1,000$; rabbit monoclonal, cat. no. 13987) antibody was purchased from Cell Signaling Technology, Inc., (Danvers, MA, USA). Anti- $\alpha$-tubulin (1:1,000; mouse monoclonal, T5168) antibody was purchased from Sigma-Aldrich; Merck KGaA. Anti-rabbit IgG, peroxidase-linked species-specific whole antibody (from donkey, NA 934; GE Healthcare) or anti-mouse IgG, peroxidase-linked species-specific whole antibody (from donkey, NA 931; GE Healthcare) were as the secondary antibody.

Cell growth and co-culture experiments. Cells were inoculated into 96-well plates at $5 \times 10^{3}$ cells per well in $0.1 \mathrm{ml}$ of DMEM supplemented with $1 \%$ D-FBS and ITH. After culture for the indicated number of days, cell growth was determined using 3-(4,5-Dimethyl-2-lythiazolyl)-2,5-diphenyl-2 $H$-tetrazolium bromide (MTT; 341-01823; Dojindo Molecular Technologies, Inc., Kumamoto, Japan) as described (14). Alternatively, cells were lysed in $10 \mathrm{mM}$ Tris- $\mathrm{HCl} \mathrm{pH} 7.4,150 \mathrm{mM} \mathrm{NaCl}, 0.9 \mathrm{mM}$ $\mathrm{CaCl}_{2}$, and $1 \%$ Triton $\mathrm{X}-100$ and growth was measured by determining GFP fluorescence intensity (excitation at $485 \mathrm{~nm}$ and emission at $538 \mathrm{~nm}$, assessed with a Cytation 5 plate reader; BioTek Instruments, Inc., Winooski, VT, USA). For co-culture experiments, Hs738 cells were first inoculated into 96-well plates at $5 \times 10^{3}$ cells/well in $0.1 \mathrm{ml}$ of DMEM supplemented with $1 \%$ D-FBS and ITH. ITV was added to the wells and the cells were cultured for 2 days. For cultivation with CM alone, culture medium from Hs 738 after 2 days of incubation with ITV was removed to new wells. Then, $10 \mu \mathrm{L}$ of a gastric cancer cell suspension $\left(5 \times 10^{4}\right.$ cells $\left./ \mathrm{ml}\right)$ in serum-free DMEM were added to the monolayer of Hs738 cells or only CM and the cells were further cultured for 3 days. For monoculture of gastric cancer cells, assay medium alone with ITV was first incubated for 2 days, and then gastric cancer cells were added as described above and further cultured for 3 days. The growth of gastric cancer cells was determined by measuring GFP fluorescence intensity as described (10). For 'non-contact' co-culture experiments, $0.6 \mathrm{ml}$ of $\mathrm{Hs} 738$ cells $\left(5 \times 10^{4}\right.$ cells $\left./ \mathrm{ml}\right)$ in DMEM supplemented with 1\% D-FBS and ITH (co-culture) or only assay medium (mono culture) were first added to outer wells of 24-well cell culture insert plates and $0.1 \mathrm{ml}$ of the assay medium was added to the inner wells with $0.4 \mu \mathrm{m}$ pore size membranes (140620; Thermo Fisher Scientific, Inc.). After 2 days of culture, $10 \mu \mathrm{L}$ of a gastric cancer cell suspension $\left(5 \times 10^{3}\right)$ in serum-free DMEM was added to the inner wells and the plates were further cultured for 3 days.

Blocking of TSP-1 activity by neutralizing antibody. Recombinant human TSP-1 $(12.5 \mu \mathrm{g} / \mathrm{ml})$ in DMEM was incubated with a monoclonal anti-TSP-1 IgM antibody (Clone A4.1 or C6.7 or not) at $37^{\circ} \mathrm{C}$ for $1 \mathrm{~h}$. MKN-74 cells were cultured at $5 \times 10^{3}$ cells $/ \mathrm{ml}$ in $0.1 \mathrm{ml}$ of above medium with ITH and dialyzed FBS in a 96-well plate for 3 days. For blocking of CM activity, CM of Hs738 was prepared with ITV $(1 \mu \mathrm{g} / \mathrm{ml})$ in the presence of $1 \%$ ITH and dialyzed FBS for 3 days. MKN-74 cells were cultured in CM or DMEM (containing 1\% ITH and dialyzed FBS) with monoclonal anti-TSP-1 IgM antibody (Clone A4.1 or C6.7 or not) for 3 days.

Statistical analysis. All data are representative of at least 3 independent experiments with similar results. Statistical analysis was carried out using two-tailed Student's t-test or analysis of variance followed by Tukey's post hoc test. $\mathrm{P}<0.05$ was considered to indicate a statistically significant difference.

\section{Results}

Effect of ITV on the growth of gastric cancer cells co-cultured with gastric stromal cells. The effect of ITV on gastric cancer cells co-cultured with gastric stromal cells was evaluated as presented in Fig. 1. The structure of ITV is shown in Fig. 1A. Human gastric cancer cells (MKN-7 and MKN-74) were cultured with human gastric stromal cells (Hs738) in the presence of various concentrations of ITV (Fig. 1C). ITV inhibited the growth of MKN-7 and MKN-74 cells cultured alone with $\mathrm{IC}_{50}$ values of 1.45 and $3.13 \mu \mathrm{g} / \mathrm{ml}$, respectively. Importantly, when co-cultured with Hs738, ITV inhibited the growth of the lines at much lower concentrations $\left(\mathrm{IC}_{50}\right.$ values of 0.12 and $0.15 \mu \mathrm{g} / \mathrm{ml}$ respectively; Fig. 1C). In contrast, the growth of Hs738 cells was inhibited by ITV treatment only when the concentration $10 \mu \mathrm{g} / \mathrm{ml}$ (Fig. 1B). MKN-7 and MKN-74 were cultured in CM taken from Hs738 cells treated with ITV (ITV CM). Growth was also inhibited more strongly than when they were cultured alone with $\mathrm{IC}_{50}$ values of 0.48 and $0.49 \mu \mathrm{g} / \mathrm{ml}$, respectively (Fig. 1C). Moreover, the growth-inhibitory activity of CM from Hs738 was dependent on the number of days with which the cells were treated with ITV (Fig. 2A and B). To clarify whether the inhibitory activity of ITV required direct cell-cell contact, we conducted 'non-contact' co-culture experiment. The data showed that ITV inhibited the growth of gastric cancer cells when they were co-cultured with Hs738 more strongly than in mono culture conditions (Fig. 2C). These results suggested that ITV induced the secretion of anti-cancer factors from Hs738 into the CM. The cytotoxicity of the highest ITV concentration $(10 \mu \mathrm{g} / \mathrm{ml})$ for Hs738, MKN-7 and MKN-74 at mono culture condition was considered to be non-specific and attributed to the high lipophilicity of ITV (Fig. 1B and C).

Determination of the anti-cancer factors in ITV CM of $H s 738$. We next prepared serum-free CM from Hs738 cells 
A<smiles>CS/C(=N\Cn1c(C/C=C(\C)CCC=C(C)C)c(C)c(=O)c2ccccc21)S(C)(C)C</smiles>

B
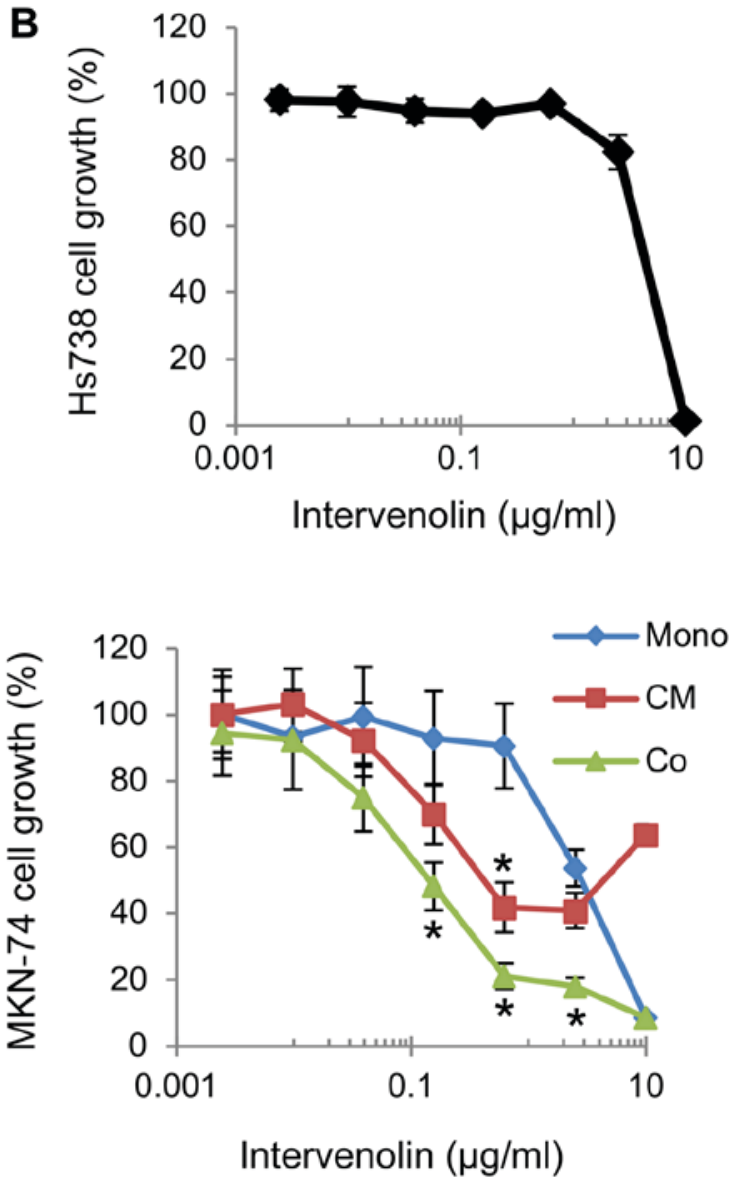

Figure 1. Activity of ITV in co-cultures of gastric cancer cells and gastric stromal cells. (A) Structure of ITV. (B) Hs738 cells were cultured for 3 days in the presence of ITV at the indicated concentrations. The growth of Hs738 cells was assessed with the MTT assay. The results shows means \pm SE ( $=3$ ). (C) MKN-7 and MKN-74 cells were cultured alone (Mono) or cultured with Hs738 (Co) for 3 days in the presence of ITV at the indicated concentrations. CM of Hs 738 cells was prepared by culturing with ITV for 2 days. Gastric cancer cell lines were cultured in the CM for 3 days (CM). Results show means \pm SE (n=3). ${ }^{*} \mathrm{P}<0.05$ vs. Mono. ITV, intervenolin; Hs738, human gastric fibroblast-like stromal cells; CM, conditioned medium.

and carried out proteomic analyses with LC-MS/MS to determine the anti-cancer factors secreted by ITV-treated HS738 cells. Whereas the growth of MKN-7 or MKN-74 cells was not changed in Ctrl CM, it was significantly inhibited in ITV CM, which was dependent on the concentration of ITV CM. This inhibitory effect of ITV CM for cancer cell growth was greater than ITV alone or Ctrl CM containing the same amount of ITV in ITV CM (Fig. 3A). Furthermore, fluorescence microscopy showed growth inhibition of MKN-7 or MKN-74 cells cultured in ITV CM. The outcome was similar to the results obtained with the MTT assay in Fig. 3A (Fig. 3B). SDS-PAGE of the CMs with silver staining showed that the ITV CM contained different proteins than did Ctrl CM, particularly around $150 \mathrm{kDa}$. These bands were excised (three bands from Ctrl CM and five bands from ITV CM; Fig. 3C). LC-MS/MS analysis revealed that these excised bands were composed of collagens, but TSP-1 was identified in band No. 5 (Score: 134, Coverage: 6.2, Mass: 133291). Because collagen band patterns were changed by ITV treatment, we demonstrated that the protein level of $\alpha$-SMA was decreased by ITV treatment (Fig. 3D). Since type I collagen and $\alpha$-SMA are myofibroblast markers, these results suggested that ITV might affect the differentiation of Hs738 cells.
ITV treatment induces TSP-1 secretion from Hs 738 cells. We further analyzed the level of TSP-1 protein in ITV CM. Western blot analysis showed the amount of TSP-1 was significantly increased by ITV treatment in serum-free $\mathrm{CM}$ that was used for proteomic analysis (Fig. 4A). We also found that TSP-1 in ITV CM increased from day 1 to day 3 of incubation (Fig. 4B). In addition, the protein level of intracellular TSP-1 in Hs738 cells was upregulated by ITV in a dose-dependent manner up to $0.5 \mu \mathrm{g} / \mathrm{ml}$ of ITV (Fig. 4C). Since the inhibitory effect of ITV CM was stronger in MKN-74 cells than MKN-7 cells (Fig. 1C), we used the former for further studies. Recombinant human TSP-1 exhibited inhibitory activity on the growth of MKN-74 cells (Fig. 4D). Semi-quantitative analysis of TSP-1 in CM by western blot using recombinant TSP-1 as control showed that ITV CM contained approximately $10 \mu \mathrm{g} / \mathrm{ml}$ of TSP-1 (Fig. 4E). In contrast, the amounts of intracellular TSP-1 in MKN-7 and MKN-74 cells were very low and they were not changed by ITV (Fig. 4F). These results suggested that ITV acts on Hs738 cells to induce the secretion of TSP-1 and that TSP-1 in CM suppresses the growth of gastric cancer cells.

Hs738 CM treated with ITV inhibits c-Myc and its downstream signaling in cancer cells. Kaur et al (15), reported that 
A

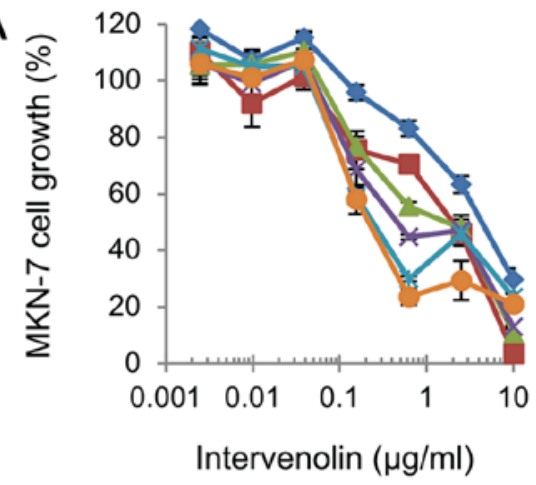

B

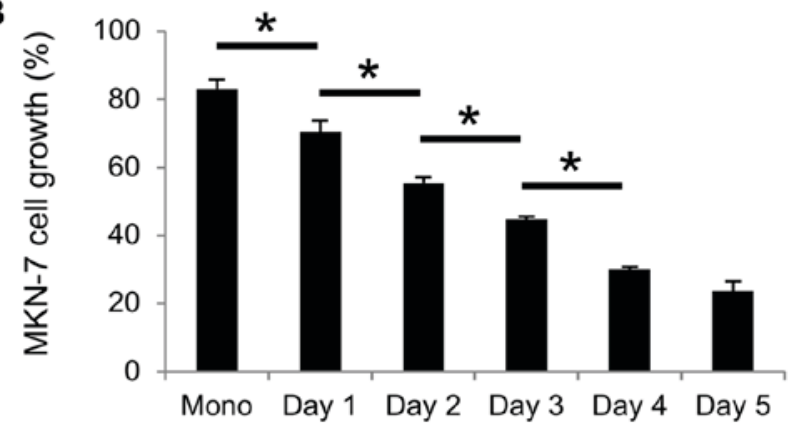

C

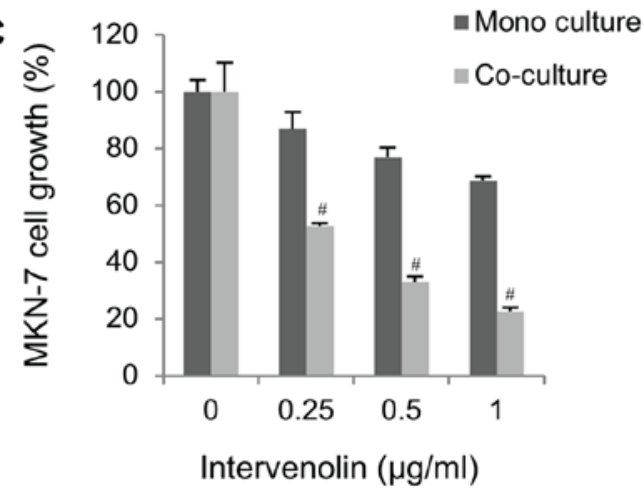

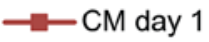

- CM day 2

$\longrightarrow$ CM day 3

* CM day 4

$\longrightarrow$ CM day 5

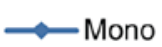

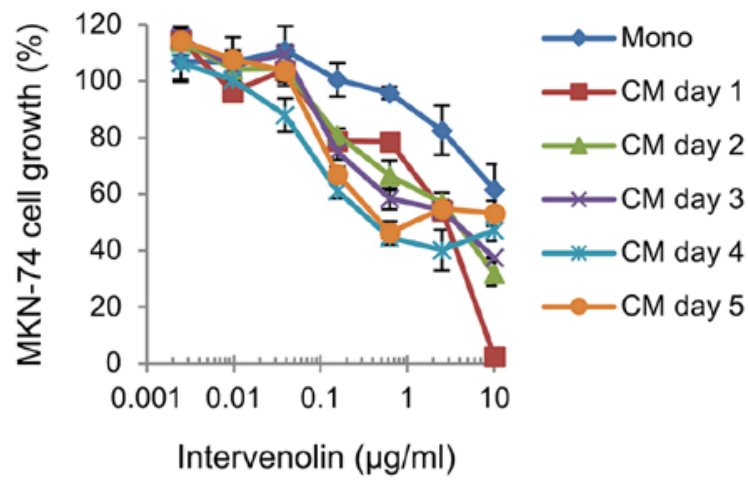

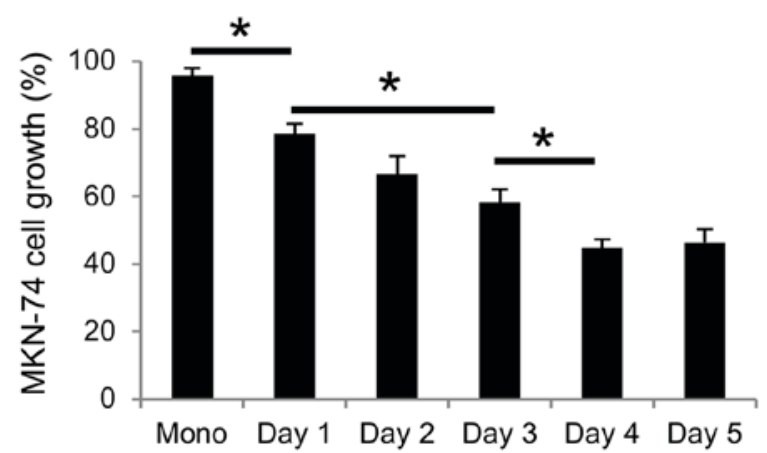

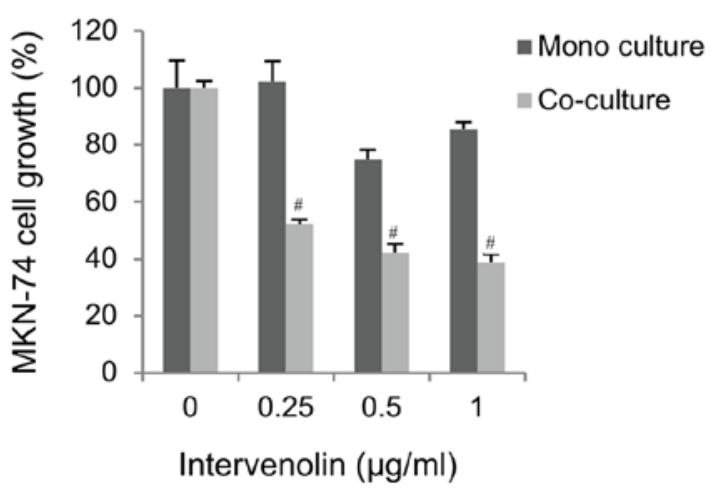

Figure 2. Growth of gastric cancer cells is inhibited by soluble factors from ITV-treated Hs738 cells. (A) CM of Hs738 cells was prepared by culturing them with ITV for the indicated number of days. Both cancer cell lines were cultured in the CMs for 3 days. Cell growth was determined by measuring GFP fluorescence intensity. (B) Cell growth of MKN-7 and MKN-74 cells in ITV CM (prepared with $0.625 \mu \mathrm{g} / \mathrm{ml}$ of ITV for indicated days) are shown. ${ }^{*} \mathrm{P}<0.05$. Results show means \pm SE $(n=3)$. Cell growth is expressed as a percentage of the value without test compounds in each culture condition. (C) MKN-7 and MKN-74 cells were cultured in inner wells of cell culture inserts for 3 days in the presence of ITV at the indicated concentrations (mono culture). For co-culture, Hs 738 cells were first cultured in outer wells of cell culture insert with indicated concentrations of ITV for 2 days, then gastric cancer cells were added to inner wells and cultured an additional 3 days (co-culture). ${ }^{\#} \mathrm{P}<0.05$ vs. Mono culture. ITV, intervenolin; Hs738, human gastric fibroblast-like stromal cells; CM, conditioned medium.

TSP-1 regulated c-Myc via CD47. Thus, we asked whether the inhibition of cancer cell growth by ITV CM was related to expression of c-Myc in cancer cells. Western blot analysis of MKN-74 cells that had been cultured in $50 \%$ or $75 \% \mathrm{CM}$ (Fig. 5A) was performed. Analysis showed that c-Myc expression in MKN-74 cells was markedly decreased by ITV CM treatment (Fig. 5A). With the suppression of c-Myc, expression levels of CDK4, whose expression is controlled by c-Myc (16), was also decreased by ITV CM. Note that the molecular weight of $\mathrm{Rb}$ protein was reduced. Those data indicated the presence of a non-phosphorylated form of this protein, suggesting that the growth of the cancer cells was likely inhibited by suppression of the cell cycle. Similar results were obtained with recombinant human TSP-1 (rTSP-1) treatment. Thus, we hypothesized that the suppression of cancer cell growth by ITV CM was due to TSP-1 that was released into the CM. It is known that the expression level of c-Myc is affected by glucose starvation (17). However, the same result was obtained even when extra glucose was added to CM (Fig. 5A).

Next, we tried to block the interaction of TSP-1 with CD36 or CD47 (receptors for TSP-1) (15), using a neutralizing antibody against each receptor. If CD47 were involved in TSP-1 signaling and cell cycle arrest, the activity of ITV CM should be cancelled by neutralizing antibody against CD47. Indeed, the growth inhibitory activity of rTSP-1 on MKN-74 cells was partially inhibited by C6.7, a neutralizing antibody for TSP-1 


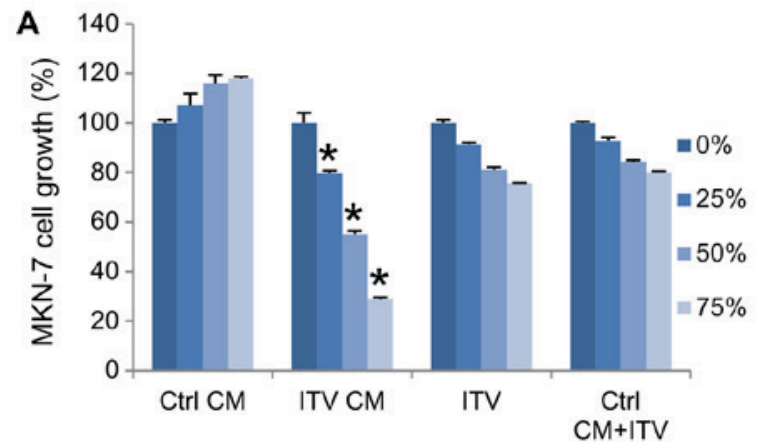

B

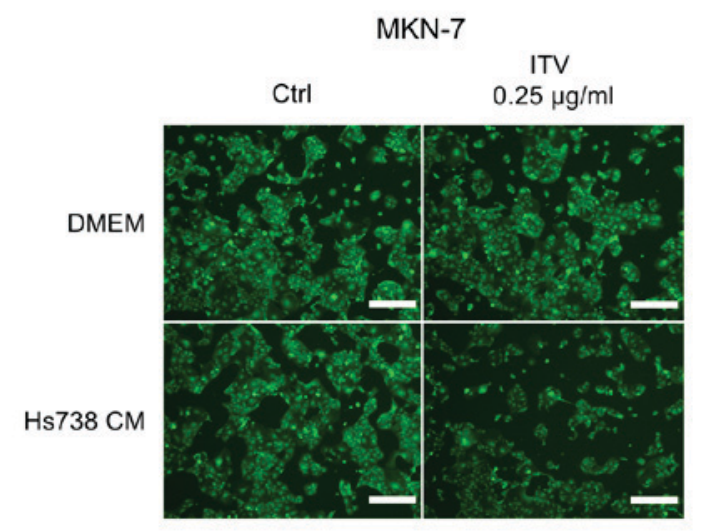

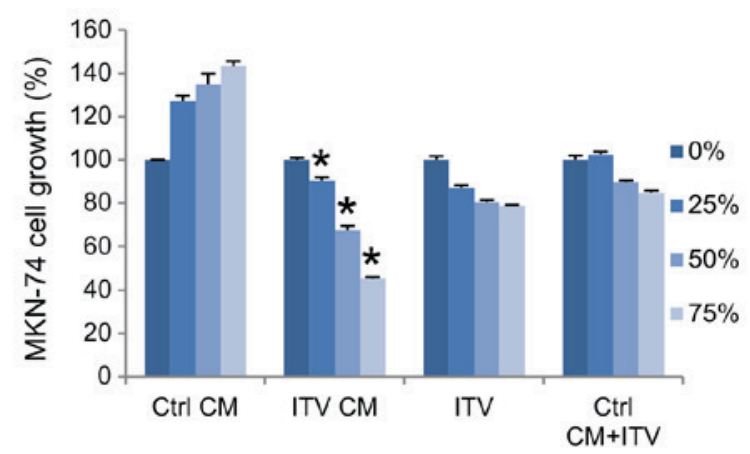

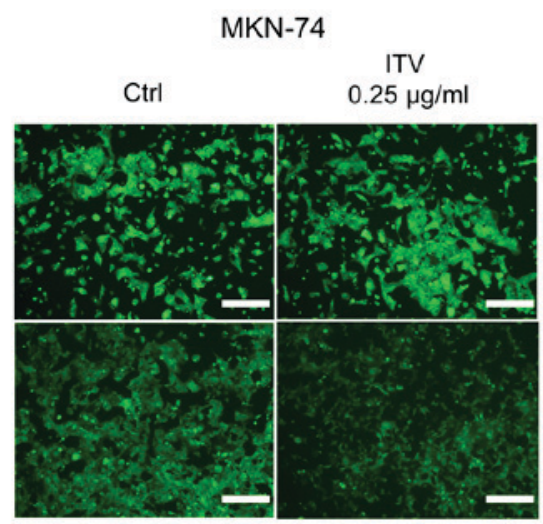

C

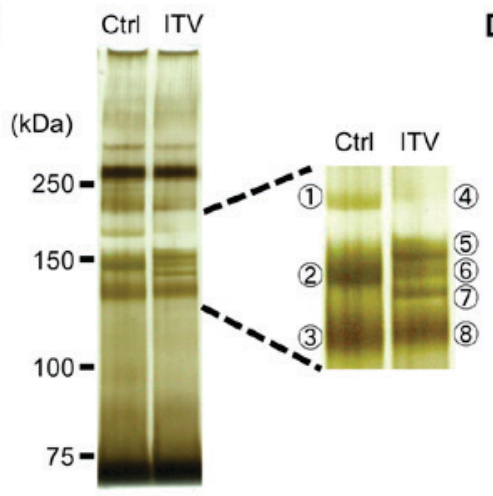

D

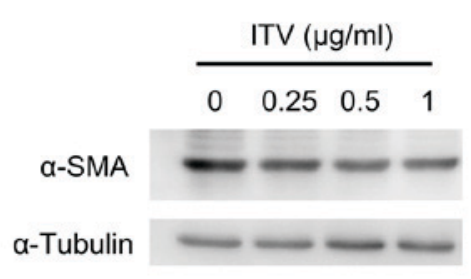

Figure 3. Determination of the anti-cancer factors in ITV CM. (A) Hs738 cells were cultured under serum-free conditions for 4 days to prepare CM. Ctrl CM, ITV CM, ITV (DMEM contained ITV at the same levels as ITV CM) or Ctrl CM+ITV (Ctrl CM contained ITV at the same amount of ITV CM) was diluted with DMEM to become 0, 25, 50, or 75\% dilution. MKN-7 or MKN-74 cells were cultured in the diluted Ctrl CM, ITV CM, ITV or Ctrl CM+ITV for 3 days. Results show means $\pm \mathrm{SE}(\mathrm{n}=3)$. ${ }^{*} \mathrm{P}<0.05$ vs. Ctrl CM. (B) Representative photomicrographs of cultured gastric cancer cell lines under fluorescence microscopy. Green: GFP. Scale bar is $200 \mu \mathrm{m}$. Upper panels show gastric cancer cell lines cultured in DMEM with or without ITV (0.25 $\mu \mathrm{g} / \mathrm{ml})$, and lower panels show both cell lines in Ctrl CM or ITV CM. (C) Equal volumes of Ctrl CM or ITV CM prepared in serum-free condition were separated with SDS-PAGE and silver-stained. The right box is an enlarged image of the stained gel and indicates excised bands. (D) Western blot analysis with extracts of Hs738 cells cultured with the indicated concentrations of ITV for 1 day. Relative intensities of determined bands of $\alpha$-SMA (normalized with $\alpha$-tubulin, analyzed by Image J) are shown. Western blot analyses were repeated at least three time with similar results. A representative result is shown here. ITV, intervenolin; CM, conditioned medium; Hs738, human gastric fibroblast-like stromal cells.

that blocks interaction with CD47. Interestingly, growth inhibition was not affected by A4.1, a neutralizing antibody that blocks interaction with CD36 (Fig. 5B). The growth inhibitory activity of ITV CM was also decreased by C6.7 (Fig. 5C). From these results, we suggest that TSP-1 in CM suppresses c-Myc via CD47 and inhibits the cell cycle of gastric cancer cells.

\section{Discussion}

In cancer chemotherapy, targeting stromal cells could be advantageous because it offers a way to circumvent the problem of tumor cell drug resistance. Moreover, the approach differs fundamentally from conventional anti-cancer drugs that directly attack the neoplastic cells. However, negative regulation of cancer cell growth by stromal cells has not been well explored. In a previous study, we found that ITV inhibited the growth of gastric cancer cell lines when they were co-cultured with Hs738, a gastric stromal cell line. The inhibition was greater than when the cells were cultured alone (11). From the results of this co-culture experiment, we suggested that ITV inhibited cancer cell growth through direct or indirect effects on the Hs738 cells. To explore this possibility, we first prepared CM of Hs738 cells treated with ITV and 
A
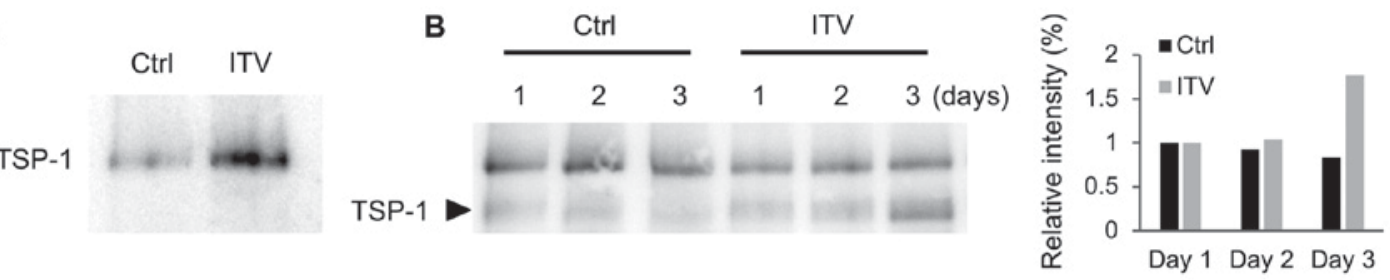

C
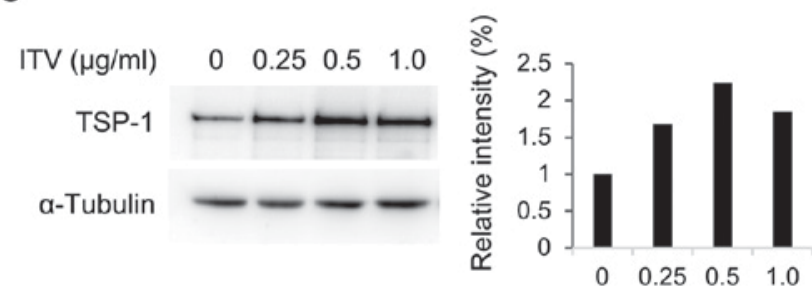

D

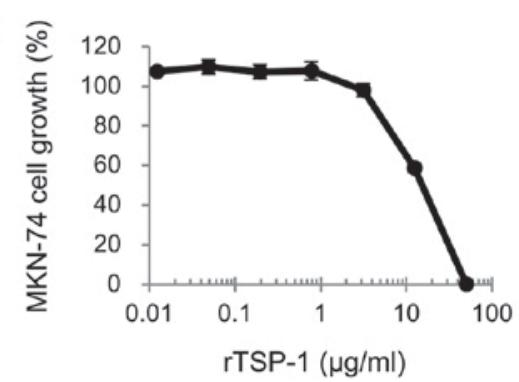

E

Recombinant human TSP-1 $(\mu \mathrm{g} / \mathrm{ml})$

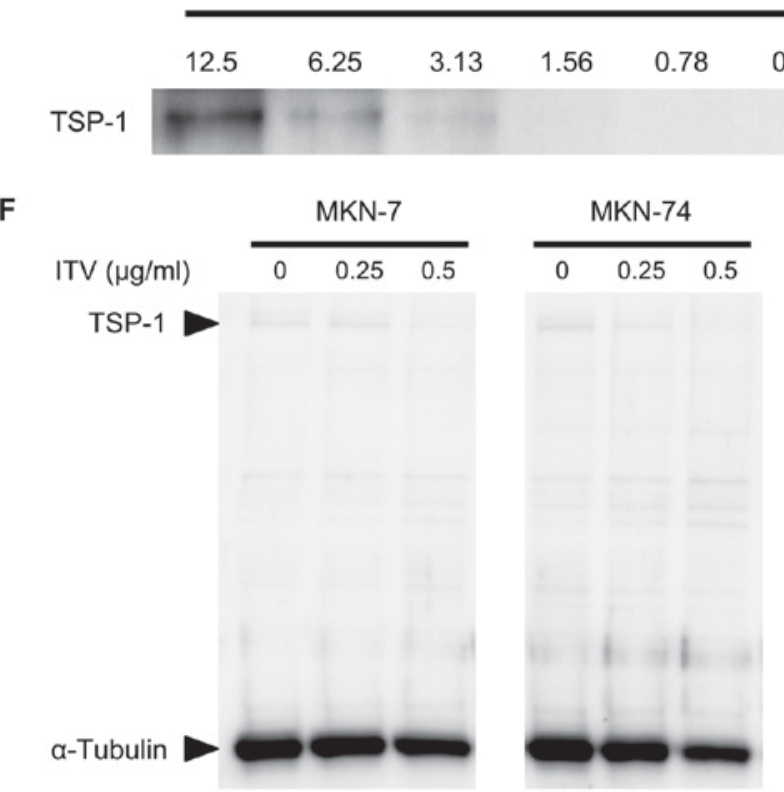

Figure 4. TSP-1 secretion from Hs738 cells is induced by ITV treatment. (A) Western blot analysis of Hs738 CM prepared under serum-free conditions by culturing for 4 days with ITV. (B) Western blot analysis of Hs738 CM. CM was prepared with or without ITV (1 $\mu \mathrm{g} / \mathrm{ml})$ in the presence of $1 \%$ dialyzed FBS and ITH for the indicated time. Equal volumes of CM were applied. Upper non-specific bands were derived from FBS. Relative intensities of indicated bands of TSP-1 are shown (analyzed by Image J). (C) Western blot analysis of extracts of Hs 738 cells cultured with the indicated concentrations of ITV for 3 days. Relative intensities of indicated bands of TSP-1 (normalized to $\alpha$-tubulin) are shown (analyzed by Image J). (D) MKN-74 cells were cultured with rTSP-1 at the indicated concentrations for 3 days. Cell growth was measured with the MTT assay. Results show means \pm SE $(n=3)$. (E) Semi-quantitative analysis by western blot using indicated concentrations of recombinant TSP-1 as control, and equal volumes of Ctrl CM or ITV CM was used. (F) Western blot analyses of extracts of MKN-7 or MKN-74 cells cultured with the indicated concentrations of ITV for 3 days. Signals of TSP-1 and $\alpha$-tubulin were determined simultaneously. Western blot analyses were repeated at least three time with similar results. A representative result is shown here. Hs738, human gastric fibroblast-like stromal cells; ITV, intervenolin; CM, conditioned medium.

examined its effect on cancer cells. We found that ITV inhibited cancer cell growth through indirect effects of Hs738 cells because ITV CM alone possessed growth inhibitory activity. Additionally, the co-culture experiments using cell culture inserts showed similar results indicating that ITV inhibited cancer cell growth through effects on Hs738 cells without direct cell-cell contact. As shown in Fig. 2A, while 1 day CM from Hs738 treated by ITV exhibited the strongest activity for inhibition of cancer cell growth, 2-5 day CM exhibited less stronger activity. For CM preparation, Hs738 cells died and burst at first 2 day culture with $10 \mu \mathrm{g} / \mathrm{ml}$ of ITV. Thus, it is considered that $\mathrm{CM}$ with $10 \mu \mathrm{g} / \mathrm{ml}$ of ITV contained very low growth inhibitory factors from Hs738 and non-specific cytotoxicity of ITV (as shown in Fig. 1A) was decreased due to unexpected interaction with some cell components from dead cells of Hs738. However, it still has non-specific cytotoxicity of ITV in Day $1 \mathrm{CM}$ because Hs738 cells are alive at Day 1 even with the highest ITV concentration. Hence, it is considered that the growth of gastric cancer cells was strongly inhibited by non-specific cytotoxicity of the highest ITV in Day $1 \mathrm{CM}$.

We next tried to identify the anti-cancer factors in CM prepared from Hs738 cells that were maintained under serum-free conditions. This approach was taken to minimize proteins from fetal bovine serum that would otherwise impair 
A

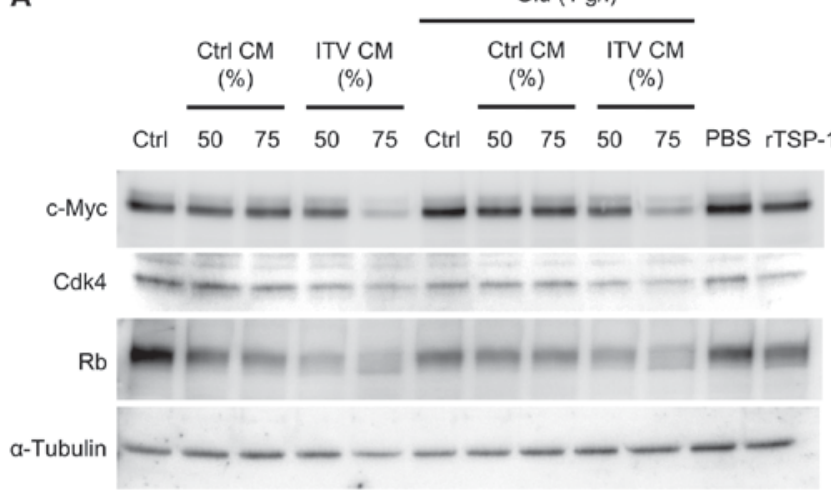

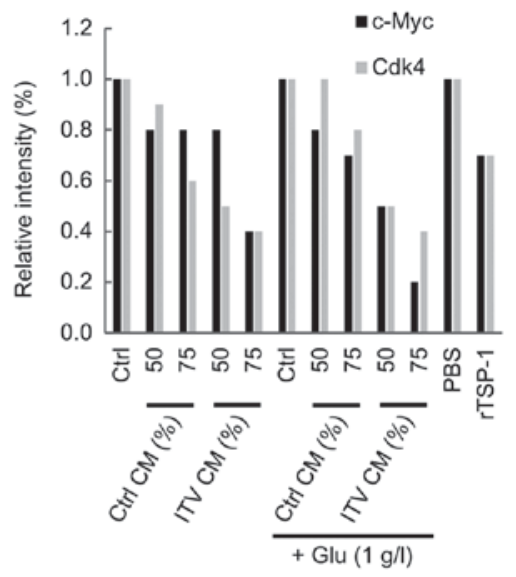

C

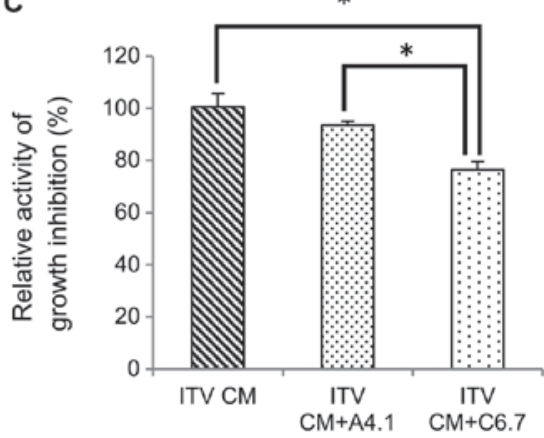

B

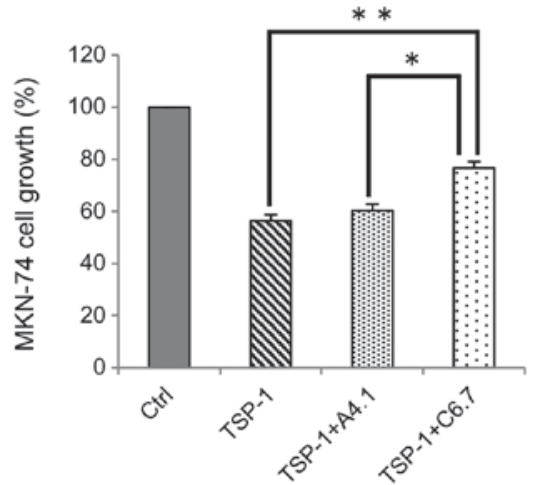

Figure 5. CM from Hs738 treated with ITV inhibits c-Myc and its downstream signaling in cancer cells. (A) Western blot analysis of extracts of MKN-74 cells that had been cultured in Ctrl CM or ITV CM at the indicated concentrations for 2 days. Glucose was supplemented into CMs at the indicated concentration (+Glu). MKN-74 cells were cultured with recombinant human TSP-1 (12.5 $\mu \mathrm{g} / \mathrm{ml}$ diluted by PBS), or DMEM with PBS (PBS). Relative intensities of designated bands (normalized to $\alpha$-tubulin, analyzed by Image $\mathrm{J}$ ) are shown. Western blot analyses were repeated at least three time with similar results. A representative result is shown here. (B) MKN-74 cells were cultured with a neutralizing antibody in the presence of recombinant human TSP-1 (12.5 $\mu \mathrm{g} / \mathrm{ml})$ for 3 days. Ctrl: DMEM; TSP-1: without antibody; TSP-1+A4.1: with antibody clone A4.1 (50 $\mu \mathrm{g} / \mathrm{ml})$; TSP-1+C6.7: with antibody clone C6.7 (50 $\mu$ g/ml). Cell growth was measured with the MTT assay. Results show means \pm SE (n=3). (C) MKN-74 cells were cultured in ITV CM or DMEM with a neutralizing antibody for 3 days. Relative activities of growth inhibition are shown. ITV CM+A4.1: ITV CM with antibody clone A4.1 (50 $\mu \mathrm{g} / \mathrm{ml})$; ITV CM+C6.7: ITV CM with antibody clone C6.7 $(50 \mu \mathrm{g} / \mathrm{ml})$. Cell growth was measured by MTT assay. Results show means $\pm \mathrm{SE}(\mathrm{n}=3)$. ${ }^{*} \mathrm{P}<0.01{ }^{* * *} \mathrm{P}<0.005$. CM, conditioned medium; Hs738, human gastric fibroblast-like stromal cells; ITV, intervenolin.

proteomic analysis. The analysis of the CM identified TSP-1 from ITV-treated stromal cells as a candidate factor. The results also revealed that type I collagen in ITV CM was decreased or its molecular weight was shifted. Additionally, we demonstrated that the protein level of $\alpha$-SMA was decreased by ITV treatment. Type I collagen and $\alpha$-SMA are cancer-associated fibroblast (CAF) markers (18). Since Hs738 cells contain a mixture of fibroblasts and myofibroblasts, this result suggested that ITV may interfere with the differentiation of Hs738 cells into the full CAF phenotype.

Kaur et al (15), reported that MYC gene expression was suppressed by TSP-1 in Jurkat cells in vitro whereas the suppression did not occur in CD47-deficient cells. Rogers et al (19), also reported that TSP-1-CD47 signaling decreased pulmonary c-Myc. The oncoprotein c-Myc directly regulates the cell cycle through transcription of its target genes, including CDK4 (17,20). Therefore, we postulated that TSP-1 in ITV CM inhibited the growth of gastric cancer cells through suppression of $M Y C$ expression and the cell cycle. Our results showed that ITV CM suppressed the expression of c-Myc protein as well as CDK4 protein in MKN-74 cells. Moreover, the growth inhibitory activities of ITV CM and rTSP-1 against MKN-74 cells were specifically blocked by neutralizing antibody against TSP-1, which inhibits the interaction with CD47. These results suggested that ITV induced TSP-1 secretion from Hs738 cells and that the secreted TSP-1 suppressed the cell cycle component in gastric cancer cells via CD47. The anti-TSP-1 neutralizing antibody did not completely block the inhibitory activity of ITV $\mathrm{CM}$. Thus, the effect of neutralizing antibody at the concentration used was a little weak because the blockade ability of the antibody against recombinant TSP-1 is equivalent to ITV $\mathrm{CM}$. However, there is also a possibility that other factors are involved in the action of ITV. Lactic acid reportedly acts as a regulator of TSP-1 expression via AP1, a transcriptional factor that binds the promoter region of THBS-1 (the TSP-1 gene), but the mode of action of ITV for induction of TSP-1 remains unknown (21) and requires further investigation.

Our data showed that ITV possesses a novel anti-cancer activity that is exerted through modulation of stromal cells. Interestingly, it has been found that TSP-1 can promote invasion, migration and proliferation of breast cancer cells (22-24). Although we demonstrated that TSP-1 negatively regulated gastric cancer cells via gastric stromal cells and that it was induced by ITV treatment, it is likely that it is the balance 
between positive and negative regulations of cancer cells that is important for TSP-1 activity.

\section{Acknowledgements}

The authors would like to thank Dr. D. Tatsuda and Mr. M. Amemiya for helpful comments, Dr. R. Sawa and Ms. Y. Takahashi for analytical measurements and Ms. C. Sakashita for technical assistance with chemical synthesis [all from Institute of Microbial Chemistry (BIKAKEN), Tokyo, Microbial Chemistry Research Foundation].

\section{Funding}

The present study was supported by JSPS KAKENHI (grant no. JP15K06888) and the Project for Cancer Research And Therapeutic Evolution (P-CREATE) from the Japan Agency for Medical Research and development, AMED (grant no. 17cm0106213h0002).

\section{Availability of data and materials}

The datasets used and/or analyzed during the present study are available from the corresponding author on reasonable request.

\section{Authors' contributions}

JY and KM designed the experiments. HA and TW synthesized and provided ITV. JY performed the study and analyzed the data. KM reviewed the manuscript. All authors read and approved the final manuscript.

\section{Ethics approval and consent to participate}

Not applicable.

\section{Patient consent for publication}

Not applicable.

\section{Competing interests}

The authors declare that they have no competing interests.

\section{References}

1. Subramaniam KS, Tham ST, Mohamed Z, Woo YL, Mat Adenan NA and Chung I: Cancer-associated fibroblasts promote proliferation of endometrial cancer cells. PLoS One 8 e68923, 2013.

2. Vermeulen L, De Sousa E Melo F, van der Heijden M, Cameron K, de Jong JH, Borovski T, Tuynman JB, Todaro M, Merz $\mathrm{C}$, Rodermond $\mathrm{H}$, et al: Wnt activity defines colon cancer stem cells and is regulated by the microenvironment. Nat Cell Biol 12: 468-476, 2010.

3. Quante M, Tu SP, Tomita H, Gonda T, Wang SS, Takashi S, Baik GH, Shibata W, Diprete B, Betz KS, et al: Bone marrow-derived myofibroblasts contribute to the mesenchymal stem cell niche and promote tumor growth. Cancer Cell 19: 257-272, 2011.

4. Malanchi I, Santamaria-Martinez A, Susanto E, Peng H, Lehr HA, Delaloye JF and Huelsken J: Interactions between cancer stem cells and their niche govern metastatic colonization. Nature 481: 85-89, 2011.
5. Wilson TR, Fridlyand J, Yan Y, Penuel E, Burton L, Chan E, Peng J, Lin E, Wang Y, Sosman J, et al: Widespread potential for growth-factor-driven resistance to anticancer kinase inhibitors. Nature 487: 505-509, 2012.

6. Kumari N, Dwarakanath BS, Das A and Bhatt AN: Role of interleukin-6 in cancer progression and therapeutic resistance. Tumour Biol 37: 11553-11572, 2016.

7. Yin SY, Jian FY, Chen YH, Chien SC, Hsieh MC, Hsiao PW Lee WH, Kuo YH and Yang N: Induction of IL-25 secretion from tumour-associated fibroblasts suppresses mammary tumour metastasis. Nat Commun 7: 11311, 2016.

8. Kawada M, Inoue H, Usami I and Ikeda D: Phthoxazolin A inhibits prostate cancer growth by modulating tumor-stromal cell interactions. Cancer Sci 100: 150-157, 2009.

9. Kawada M, Inoue H, Ohba S, Masuda T, Momose I and Ikeda D: Leucinostatin A inhibits prostate cancer growth through reduction of insulin-like growth factor-I expression in prostate stromal cells. Int J Cancer 126: 810-818, 2010.

10. Kawada M, Inoue H, Ohba S, Yoshida J, Masuda T, Yamasaki M, Usami I, Sakamoto S, Abe H, Watanabe T, et al: Stromal cells positively and negatively modulate the growth of cancer cells: Stimulation via the PGE2-TNFa-IL-6 pathway and inhibition via secreted GAPDH-E-cadherin interaction. PLoS One 10: e0119415, 2015.

11. Kawada M, Inoue $\mathrm{H}$, Ohba $\mathrm{S}$, Hatano M, Amemiya M, Hayashi $\mathrm{C}$, Usami I, Abe H, Watanabe T, Kinoshita N, et al: Intervenolin, a new antitumor compound with anti-Helicobacter pylori activity, from Nocardia sp. M196-86F2. J Antibiot (Tokyo) 66: 543-548, 2013.

12. Rodriguez-Manzaneque JC, Lane TF, Ortega MA, Hynes RO, Lawler J and Iruela-Arispe Ml: Thrombospondin-1 suppresses spontaneous tumor growth and inhibits activation of matrix metalloproteinase- 9 and mobilization of vascular endothelial growth factor. Proc Natl Acad Sci USA 98: 12485-12490, 2001.

13. Kawada M, Masuda T, Ishizuka $M$ and Takeuchi $T$ : 15-Deoxyspergualin inhibits Akt kinase activation and phosphatidylcholine synthesis. J Biol Chem 277: 27765-27771, 2002.

14. Fukazawa H, Mizuno S and Uehara Y: A microplate assay for quantitation of anchorage-independent growth of transformed cells. Anal Biochem 228: 83-90, 1995.

15. Kaur S, Soto-Pantoja DR, Stein EV, Liu C, Elkahloun AG, Pendrak Ml, Nicolae A, Singh SP, Nie Z, Levens D, et al: Thrombospondin-1 signaling through CD47 inhibits self-renewal by regulating c-Myc and other stem cell transcription factors. Sci Rep 3: 1673, 2013.

16. Miliani de Marval PL, Macias E, Rounbehler R, Sicinski P, Kiyokawa H, Johnson DG, Conti CJ and Rodriguez-Puebla Ml: Lack of cyclin-dependent kinase 4 inhibits c-myc tumorigenic activities in epithelial tissues. Mol Cell Biol 24: 7538-7547, 2004.

17. Wu S, Yin X, Fang X, Zheng J, Li L, Liu X and Chu L: c-MYC responds to glucose deprivation in a cell-type-dependent manner. Cell Death Discov 1: 15057, 2015.

18. Togo S, Polanska UM, Horimoto $\mathrm{Y}$ and Orimo A: Carcinoma-associated fibroblasts are a promising therapeutic target. Cancers 5: 149-169, 2013.

19. Rogers NM, Sharifi-Sanjani M, Yao M, Ghimire K, Bienes-Martinez R, Mutchler SM, Knupp HE, Baust J, Novelli EM, Ross M, et al: TSP1-CD47 signaling is upregulated in clinical pulmonary hypertension and contributes to pulmonary arterial vasculopathy and dysfunction. Cardiovasc Res 113: 15-29, 2017.

20. Schmidt EV: The role of c-myc in cellular growth control. Oncogene 18: 2988-2996, 1999.

21. Seliger C, Leukel P, Moeckel S, Jachnik B, Lottaz C, Kreutz M, Brawanski A, Proescholdt M, Bogdahn U, Bosserhoff AK, et al: Lactate-modulated induction of THBS-1 activates transforming growth factor (TGF)-beta2 and migration of glioma cells in vitro. PLoS One 8: e78935, 2013.

22. Albo D, Rothman VL, Roberts DD and Tuszynski GP: Tumour cell thrombospondin-1 regulates tumour cell adhesion and invasion through the urokinase plasminogen activator receptor. $\mathrm{Br}$ J Cancer 83: 298-306, 2000.

23. Hyder SM, Liang Y and Wu J: Estrogen regulation of thrombospondin-1 in human breast cancer cells. Int J Cancer 125: 1045-1053, 2009.

24. Yee KO,Connolly CM,Duquette M,Kazerounian S, Washington R and Lawler J: The effect of thrombospondin-1 on breast cancer metastasis. Breast Cancer Res Treat 114: 85-96, 2009. 